\title{
Composite Method for Image Retrieval
}

\author{
D. Madhavi, N. Jyothi, Md. K. M. Chisti
}

\begin{abstract}
In the present day to day life the retrieval of images from the larger database has become important like Google search. Presently, CBIR is trending method. In this paper images are retrieved in three stages with color, texture and shape feature extracted in each respective stage. The color feature extraction is done through finding different parameters for an RGB image, combined texture feature extraction through Gray level co-occurrence matrix GLCM and Gabor filter and shape feature extraction through region growing. The experiment is done on images present in coral database. The simulation results have been compared and it has been shown that the proposed method show high retrieval rate in terms of the average precision and average recall.
\end{abstract}

Keywords : Image Retrieval, Gray level co-occurrence matrix (GLCM), Region growing.

\section{INTRODUCTION}

Content based image retrieval deals with retrieval of images that rely on image contents. Image contents can be obtained using three low level features [1] details extracted from an image. A color histogram based image retrieval is proposed by [2]. GLCM features [3] used in existing retrieval systems are shown to be having low average recall and average precision rate. This can be used further improved using hybrid methods [4]. Content based image retrieval (CBIR) also known as query by image and video content (QBIC) is basically used to search digital images present in large databases. Database used in this paper is Corel database [6]. Corel database comprises collection of 1000 images with 100 images in each category. CBIR technique retrieves images faster than text based image retrieval. In text based image retrieval, images are retrieved by giving name of the image. By doing this the accurate results are not obtained whereas in content based image retrieval the features of the image are computed and are compared with database images which effectively retrieve the images. CBIR has been used by many researchers in various fields of medical, military, biodiversity, digital libraries and commercial systems[5]. Gabor filters for texture defect detection is employed for locating accurate defect position [11].

Revised Manuscript Received on February 05, 2020.

* Correspondence Author

D. Madhavi*, Associate professor, Department of EECE, GITAM Deemed to

University,Visakhapatnam,India,E.Mail:madhavi.dunna@gitam.edu.

N. Jyothi, Associate professor, Department of EECE, GITAM Deemed to be University,Visakhapatnam,India,E.Mail:jyothi.nutakki@gitam.edu.

M. D. K. M. Chisti, Assistant professor, Department of EECE, GITAM Deemed to be University,Visakhapatnam, India, E.Mail: mohammedkm.chisti@gitam.edu.

(C) The Authors. Published by Blue Eyes Intelligence Engineering and Sciences Publication (BEIESP). This is an open access article under the CC BY-NC-ND license (http://creativecommons.org/licenses/by-nc-nd/4.0/)

\section{PROPOSED METHOD}

This section describes the step by step procedure adopted for the image retrieval system.

The block illustration of the retrieval system is as displayed in the Figure 1.

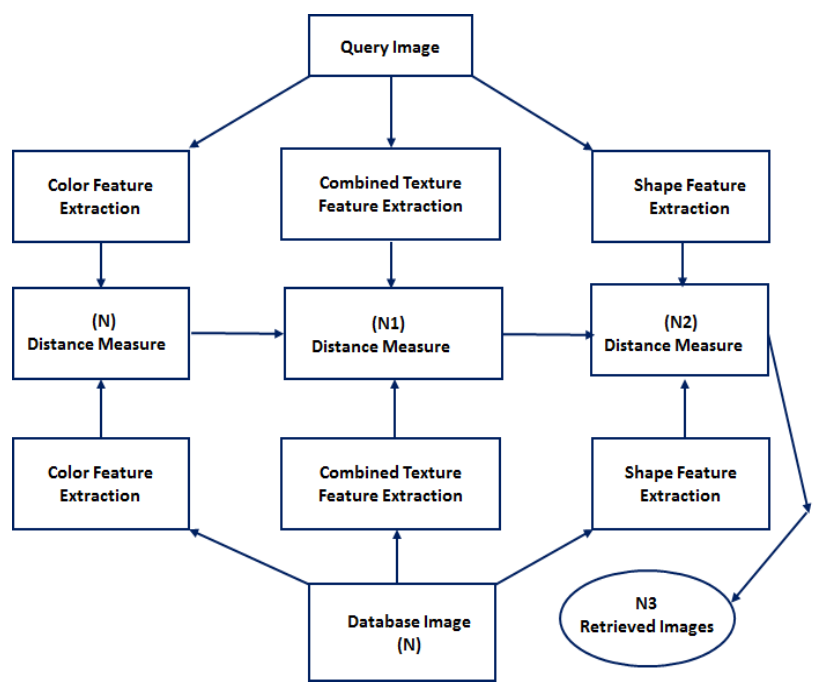

Fig. 1.Block Illustration of proposed method showing three stages of retrieval system

Initially, calculate color feature for query image. Color feature constitutes mean, variance and standard deviation [9]. Next calculate same features for every database image. The difference between features is calculated with Euclidean distance.

The least distance between images is considered as best matched images and are retrieved. Considering $\mathrm{N}$ database images, N1 images are retrieved basing on color features, where $(\mathrm{N} 1<\mathrm{N})$ and these $\mathrm{N} 1$ images are considered as input database images for the next stages of retrieval system. Likewise, in the next stage, texture features are computed for the N1 metaphors. The best matched N2 metaphors are extracted from $\mathrm{N} 1$ images using same distance measure $(\mathrm{N} 2<\mathrm{N} 1)$. Texture feature extraction is pronounced in detail in next section. Finally, shape features are extracted on the images retrieved in the above stage. The best match images N3 are retrieved in similar manner. Therefore, at each stage maintaining the condition $\mathrm{N} 3<\mathrm{N} 2<\mathrm{N} 1<\mathrm{N}$ images are retrieved. The details of each stage and the corresponding distance measure are presented in the following sections.

\section{HYBRID RETRIEVAL SYSTEM}

This section pronounces about three successive stages of image retrieval system. The stages are based on color, combined texture and shape features successively.

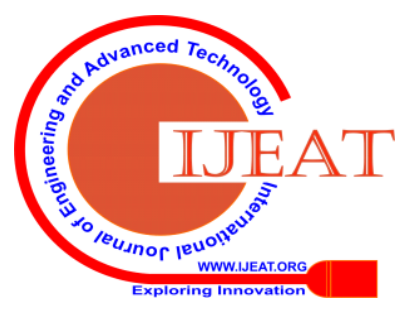


Figure 2 shows one image from each category of images of the database and Figure 3 shows the randomly selected query image.

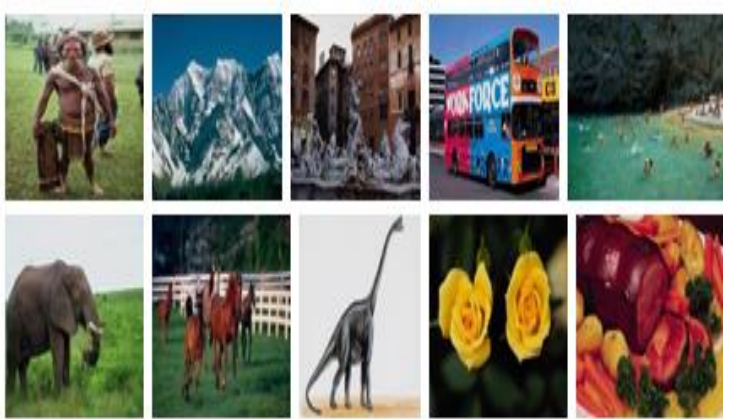

Fig. 2.One image from each group of images from the Corel database

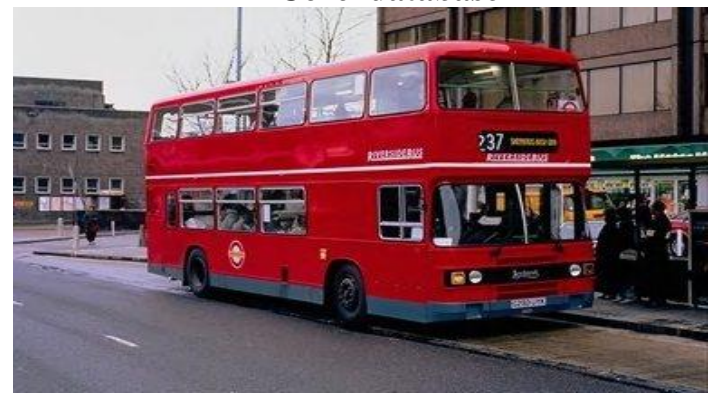

Fig. 3.Query image

\section{A. Stage 1}

In this stage, color feature extraction is performed. Database images are considered to be RGB images. First calculation of mean is done for each frame of an RGB image. Mean of R frame (FR), G frame (FG) and B frame (FB) are calculated respectively using the expressions as given from Eq. 1 to Eq.3 and average of the three means is computed (FM) using Eq.4. The mean (FM) is calculated for query image and later for every database image. The parameters variance as well as standard deviation are calculated using Eq.5 and Eq.6. If the Euclidean distance between query and each database image $(\mathrm{N})$ is computed, then the least distanceN1 images are retrieved. This is done by sorting the obtained distances and choosing the first $\mathrm{N} 1$ images $(\mathrm{N} 1<\mathrm{N})$. Finally, retrieved images are displayed as shown in the Figure 4.

$F_{R}=\sum_{i=1}^{p} \sum_{j=1}^{q} \frac{m_{i j}}{p q}$

$F_{G}=\sum_{i=1}^{p} \sum_{j=1}^{q} \frac{m_{i j}}{p q}$

$F_{B}=\sum_{i=1}^{p} \sum_{j=1}^{q} \frac{m_{i j}}{p q}$

$F_{M}=\frac{F_{R}+F_{G}+F_{B}}{3}$

Variance $\mathrm{V}=\frac{1}{p q} \sum_{i=1}^{p} \sum_{j=1}^{q}\left(m_{i j}-\mathrm{F}_{\mathrm{M}}\right)^{2}$

Standard deviation $\mathrm{D}=\sqrt{V}$

Where $m_{i j}$ is pixel value of the $\mathrm{i}^{\text {th }}$ row and $\mathrm{j}^{\text {th }}$ column.
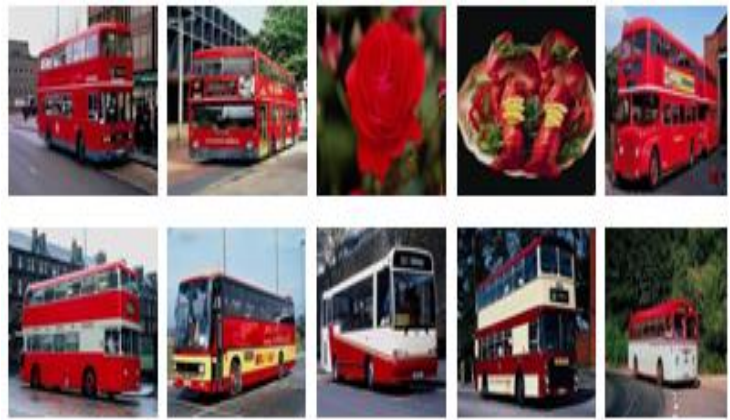

Fig. 4.Images extracted using color features

\section{B. Stage 2}

In this stage, texture features are computed for the images retrieved in the stage 1 . In this stage images of the previous stage are examined with the texture features employing the combined GLCM and Gabor Filter features [1]. In this section, feature extraction is explained with necessary computational equations.

\section{- GLCM Features}

Gray level co-occurrence matrix is used for the texture feature calculation [7]. There exist numerous GLCM texture features. In this paper eight GLCM features are selected as they have shown much variation between the dissimilar groups of images. This is concluded by finding the histogram of twenty-five second order GLCM features and the most representative features suitable for Corel database are being selected. For the computation, initially the color image is transformed to gray image and then GLCM matrix is computed. GLCM gives the joint probability of the occurrence of the two pair of pixels at a distance d. But, in this paper one-pixel distance is considered. GLCM features [3] are extracted for both query and retrieved N1 database images. In this work, GLCM features such as correlation, energy, homogeneity, image entropy, contrast, sum average, sum variance and difference variance are considered and computed using Eq.7 to Eq.14 respectively.

Correlation $=\sum_{\mathrm{i}, \mathrm{j}} \frac{\left(\mathrm{i}-\mathrm{m}_{\mathrm{i}}\right)\left(\mathrm{j}-\mathrm{m}_{\mathrm{j}}\right) p_{i j}}{\sigma_{i} \sigma_{\mathrm{j}}}$

Energy $=-\sum_{i} \sum_{j} p^{2}(i, j)$

Homogeneity $=\sum_{i} \sum_{j} \frac{p_{(i, j)}}{1+|i-j|}$

Image Entropy $=-\sum_{i} \sum_{j} p(i, j) \log (p(i, j))$

Contrast $=\sum_{n=0}^{N_{g}-1} n^{2}\left\{\sum_{i=1}^{N_{g}} \sum_{j=1}^{N_{g}} p(i, j)\right\}|i-j|$

Sum average $=\sum_{I=2}^{2 N_{g}} i p_{x+y}(i)$

Sum variance $=\sum_{i=2}^{2 N_{g}}\left(i-f_{S}\right)^{2} p_{x+y}(i)$

Difference variance $=\sum_{i=0}^{N_{g}-1} i^{2} p_{x+y}(i)$

\section{- Gabor Filter Features}

2D Gabor filter is widely employed in texture feature extraction as it is most subtle to the texture variations in the image [12]. Gabor mask over the image can transform image into the image feature. In the proposed system a gray Gabor filter given in Eq. 15 is employed with fixed 60 x 60 mask size.

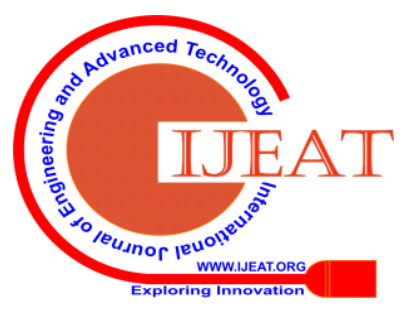


$F(x, y, \theta, \omega, \sigma)=\frac{1}{2 \pi \sigma^{2}} e^{\left(-\frac{1}{2}\left(\frac{x^{2}+y^{2}}{\sigma^{2}}\right)+j \omega(x \cos \theta+y \sin \theta)\right)}$

Where the parameters are chosen as spatial scale $\sigma=60$ and frequency $\omega=15$ and orientation $\theta$ as $0^{\circ}, 45^{\circ}, 90^{\circ}, 135^{\circ}$.

This predesigned filter banks are convolved with the original image and results in a complex quantity. The energy $\mathrm{G}$ of the Gabor filter is computed by taking square sum of the real and imaginary components of the obtained convolution result.

$G(i)=\operatorname{Re}\{I * F\}^{2}+\operatorname{Im}\{I * F\}^{2}$

Where $\mathrm{i}=0$ to 3 for four different orientations, * represents complex convolution and $\mathrm{F}$ is Gabor filter function obtained from Eq. 15.

The set of equations from Eq. 7 to Eq. 15 are used as combined feature set for the texture feature retrieval in the second stage. The retrieved images obtained at this stage are displayed in Figure 5.
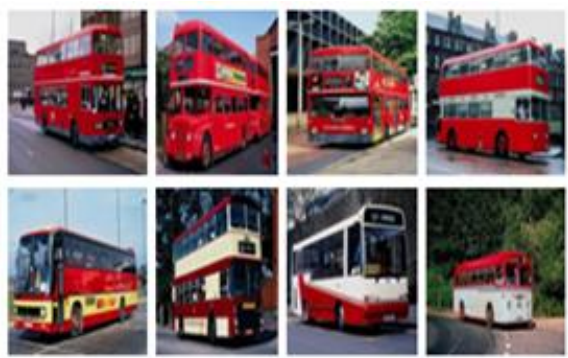

Fig. 5.Images extracted using texture features

\section{Stage 3}

In third stage, shape feature extraction is performed. Shape feature is computed using region growing algorithm [8]. Initial seed points are estimated/randomly selected. Seed points are selected from the white regions of the image by converting the input image into black and white. Region growing explains that pixels which are close together have similar gray values. For both query and database image boundaries are calculated and compared. If the distance between them is minimum, the images are retrieved. Area is represented by A, Bounding box Area by B, convex area by $\mathrm{C}$ and perimeter is represented by $P$. In this paper, shape features are extracted making use of features such as extent, equivalence diameter, circularity and solidity and they are calculated using equations Eq.17 to Eq.20.

Extent $=\frac{A}{B} \pi$

Equivalence Diameter $=\operatorname{sqrt}\left(\frac{4^{*} A}{\pi}\right)$

Circularity $=4 \pi\left(\frac{A}{P}\right)$

Solidity $=\frac{A}{C}$

The N3 images are retrieved by treating N2 images obtained in stage 2 as input database and are displayed in Figure 6.
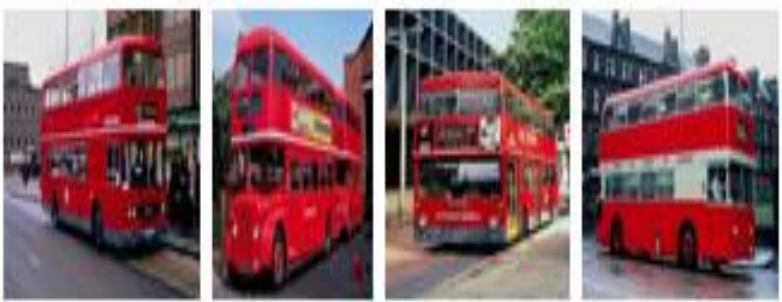

Fig. 6.Images extracted using shape features

\section{SYSTEM EVALUATION}

The proposed system is evaluated using similarity measure at each stage based on Euclidean distance [E] between the features. This can be computed usingEq.21.

$\mathrm{E}=\sqrt{\left(m_{q}-m_{d}\right)^{2}+\left(v_{q}-v_{d}\right)^{2}+\left(s_{q}-s_{d}\right)^{2}}$

The distance measure is performed and the nearest match images are selected for the next stage at each stage and in the last stage, retrieved images are used to evaluateperformance of system based on $\alpha$ and $\beta$ as given in Eq.22 and Eq.23.

Precision $\alpha=\frac{\text { No.of relevant images retrieved }}{\text { Total } \text { No.of images retrieved }}$

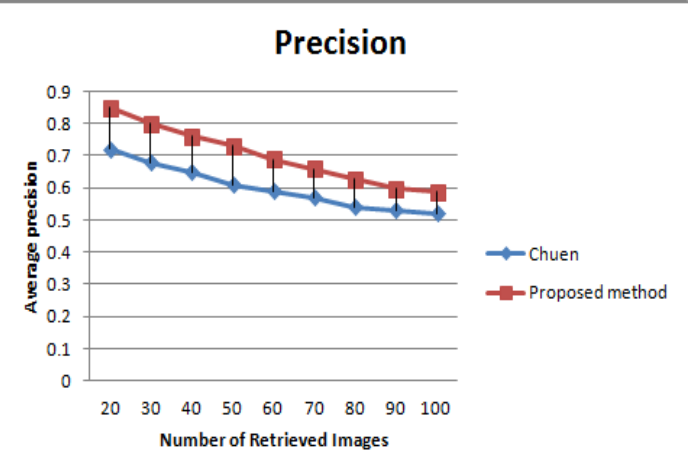

Fig. 7.Graph for comparison of average precision with respect to the database image sets

Recall $\beta=\frac{\text { No.of relevant images retrieved }}{\text { Total No.of relevant images in the database }}$

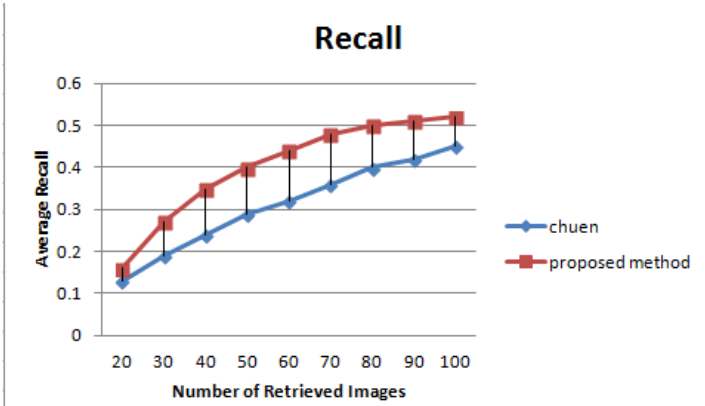

Fig. 8.Graph for comparison of average recall with respect to the database image sets

Figure 7 and Figure 8 shows the system performance measured against performance metrics $\alpha$ and $\beta$ in regard to the number of retrieved metaphors. Table 1 and Table 2 has shown the experimental results of proposed method with existing system in terms of average precision and average recall.

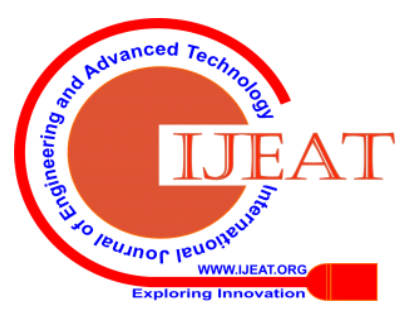


Table- I: Comparison of average precision with respect to database image sets

\begin{tabular}{|c|c|c|c|}
\hline Category & Class & Proposed method & $\begin{array}{c}\text { Chuen } \\
{[\mathbf{1 0}]}\end{array}$ \\
\hline 1 & Tribal & 0.85 & 0.72 \\
\hline 2 & Mountains & 0.8 & 0.68 \\
\hline 3 & Buildings & 0.76 & 0.65 \\
\hline 4 & Buses & 0.73 & 0.61 \\
\hline 5 & Beaches & 0.71 & 0.67 \\
\hline 6 & Elephants & 0.69 & 0.59 \\
\hline 7 & Horses & 0.66 & 0.57 \\
\hline 8 & Dinosaurs & 0.63 & 0.54 \\
\hline 9 & Flowers & 0.6 & 0.53 \\
\hline 10 & Food & 0.59 & 0.52 \\
\hline \multicolumn{2}{|c|}{ Average } & 0.7 & 0.6 \\
\hline
\end{tabular}

Table- II: Comparison of average recall with respect to the database image sets

\begin{tabular}{|c|c|c|c|}
\hline Category & Class & Proposed method & $\begin{array}{c}\text { Chuen } \\
{[\mathbf{1 0}]}\end{array}$ \\
\hline 1 & Tribal & 0.16 & 0.13 \\
\hline 2 & Mountains & 0.27 & 0.19 \\
\hline 3 & Buildings & 0.35 & 0.24 \\
\hline 4 & Buses & 0.4 & 0.29 \\
\hline 5 & Beaches & 0.42 & 0.34 \\
\hline 6 & Elephants & 0.44 & 0.32 \\
\hline 7 & Horses & 0.48 & 0.36 \\
\hline 8 & Dinosaurs & 0.5 & 0.4 \\
\hline 9 & Flowers & 0.51 & 0.42 \\
\hline 10 & Food & 0.52 & 0.45 \\
\hline \multicolumn{2}{|c|}{ Average } & 0.4 & 0.3 \\
\hline
\end{tabular}

\section{CONCLUSION}

In this paper, a hybrid method for image retrieval based on three different features is proposed. The features are extracted by cascading three stages. The results revealed that cascading three stages has shown better retrieval rate when compared to the existing method in terms of average precision as well as average recall. The texture featureschosen in this paper are a combination ofGLCM and Gabor filter features which are closely examined specific to the Corel database that influenced the retrieval rate. This work can be extended further by training feature set with artificial neural networks thatmay result in better performance metrics.

\section{REFERENCES}

1. D.Madhavi, M.R.Patnaik, "Image Retrieval using GA Optimized Gabor Filter,'Indian Journal of Science and Techonology, vol .9,no.44, 2016,pp.1-11.

2. C.A.Hussain, D.V. Rao, T.Praveen, "Color Histogram based Image Retrieval,” International Journal of Advanced Engineering Technology, Sept.2013,pp.63-66.

3. https://in.mathworks.com/matlabcentral/fileexchange/22187-glcm-text ure-features.

4. D.Madhavi, K. M. C Mohammed, N.Jyothi ,M.R. Patnaik, "A hybrid content based image retrieval systemusing log-gabor filter banks," International Journal of Electrical and Computer Engineering, vol. 9, No. 1,Feb. 2019,pp.237-244.

5. N.Jyothi, D.Madhavi, "PSO Optimized Log Gabor QBIC System," International Journal of Innovative Technology and Exploring Engineering,vol.8, Issue-11, Sept. 2019.

6. http://wang.ist.psu.edu/docs/related.

7. Benco,M., R. Hudec, P. Kamencay, M. Zachariasova and S. Matuska, "An Advanced Approach to Extraction of Colour Texture Features
Based on GLCM," International Journal of Advanced Robotic Systems, vol. 11, issue. 7, May 2014.

8. Yue, J., Z. Li, L. Liu and Z. Fu, "Content-based image retrieval using color and texture fused features," Mathematical and Computer Modelling, vol. 54, Issues 3-4, pp. 1121-1127, Aug. 2011.

9. R.Gonzalez, R.woods, Digital Image Processing, Addison-Wesley Publishing, $4^{\text {th }}$ edn.,2018.

10. L.T Chuen, C. C. Chen, "Color Image Retrieval Technique Based on Color Featuresand Image Bit Map,” Inf Process Manage, 43,2007, pp.461-472.

11. K. M. C Mohammed, S.S Kumar, G.Prasad,"2D Gabor Filter for Surface Defect Detection Using GA and PSO Optimization Techniques," ASME Journals Series: Advances B; vol. 58, 2015, pp-67-83.

12. D.Madhavi, M.R. Patnaik, "Image Retrieval Based on Tuned Color Gabor Filter Using Genetic Algorithm,’ International Journal of Applied Engineering Research, vol. 12, no.15, 2017, pp. 5031-5039.

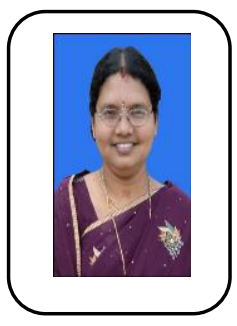

\section{AUTHORS PROFILE}

D.Madhavi has done Ph.D. from Andhra University in 2018,M.Tech.from Andhra University in 2004,A.M.I.E in 2000.She secured Suman Sharma award for highest total in A.M.I.E. She is having 19 years of teaching and research experience She is an Associate Professor in GITAM Deemed to be University, Visakhapatnam. Her areas of interest include image processing, VLSI, signal processing and neural networks

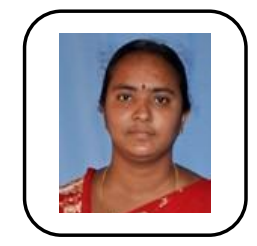

N.Jyothi has done Ph.D from Andhra University in 2019, M.E from Andhra University in 2003, B.Tech. from Nagarjuna University in 2001. She is having 17 years of teaching and research experience. She is an Associate Professor in GITAM Deemed to be University, Visakhapatnam. Her areas of interest include signal processing, VLSI and image processing.

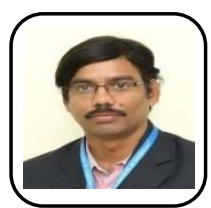

Md. Khwaja Muinuddin Chisti, is a senior member of IEEE and presently working as an Assistant Professor in EECE, GITAM Deemed to be University, Visakhapatnam. He studied his Masters from Andhra University, Visakhapatnam and B.Tech. in Electronics and Communication Engineering from Aditya Engineering College, Kakinada, India. His research areas are Image processing, Machine Learning, Deep Learning, Embedded Systems, and Signal Processing. 